\title{
Cerebral microbleeds in neurological practice: concepts, diagnostics and clinical aspects
}

\author{
Aleksandra Wach-Klink ${ }^{1}$, Ewa Iżycka-Świeszewska ${ }^{2,3}$ (D) Grzegorz Kozera ${ }^{4}$, Piotr Sobolewski ${ }^{1,5}$ (1) \\ ${ }^{1}$ Department of Neurology and Stroke Unit in Sandomierz, Jan Kochanowski University, Kielce, Poland \\ ${ }^{2}$ Department of Pathology and Neuropathology, Medical University of Gdansk, Poland \\ ${ }^{3}$ Department of Pathomorphology, Copernicus Hospitals Gdansk, Poland \\ ${ }^{4}$ Medical Simulation Centre, Medical University of Gdansk, Poland \\ ${ }^{5}$ Collegium Medicum, Jan Kochanowski University, Kielce, Poland
}

\begin{abstract}
Introduction: Due to the widespread use of magnetic resonance imaging (MRI) in neurological diagnostics, the number of patients detected as having cerebral microbleeds (CMBs) continues to increase. However, their clinical impact still remains controversial, especially the question of whether CMBs significantly increase the risk of life-threatening intracerebral haemorrhage (ICH) in patients undergoing intravenous thrombolysis (IVT) or endovascular thrombectomy (EVT), or in patients on anticoagulant therapy or statins.

State of the art: The term 'CMB' is a radiological concept that aims to illustrate microscopic pathology of perivascular hemosiderin deposits corresponding most probably to small foci of past bleeding. MRI images in sequence T2*-GRE and susceptibility-weighted imaging (SWI) are used for a diagnosis of a CMB. This review summarises the current knowledge regarding the definition, prevalence, genetics, risk factors, radiological diagnosis and differential diagnosis of a CMB. We discuss its role as an indicator of future ischaemic or haemorrhagic events in high risk patients or those on antiplatelet or anticoagulant therapy, and its prognostic value for reperfusion strategies and for the development of dementia.

Future direction: The place of CMBs in current guidelines is explored herein. It must be emphasised that the recommendations relating to CMBs are expert opinions. Therefore, at the end of this review, we pose a number of questions that future clinical trials should answer.
\end{abstract}

Key words: cerebral microbleed, small vessel disease, intravenous thrombolysis, endovascular thrombectomy, antithrombotic therapy (Neurol Neurochir Pol 2021; 55 (5): 450-461)

\section{Introduction}

Due to the ever-increasing use of head magnetic resonance imaging (MRI), the population of patients being diagnosed with cerebral microbleeds (CMBs) continues to increase $[1,2]$. The term CMB is a radiological concept that aims to illustrate microscopic pathology of perivascular hemosiderin deposits corresponding to small foci of past bleeding. The detection of $\mathrm{CMBs}$ in neuroimaging mainly concerns the older population, patients with previous haemorrhagic and ischaemic strokes, patients with various types of dementia, patients with neurogenerative diseases of the nervous system, and patients with hypertension and amyloid angiopathy [2-4].

The most important clinical question is whether CMBs increase the risk of a life-threatening intracerebral haemorrhage (ICH), especially in patients undergoing intravenous thrombolysis (IVT), endovascular thrombectomy (EVT) or who are being treated with anticoagulants or statins. However,

Address for correspondence: Aleksandra Wach-Klink, Department of Neurology and Stroke Unit of UJK and of Holy Spirit Specialist Hospital in Sandomierz, 13 Schinzla Str., 27-600 Sandomierz, Kielce, Poland; e-mail: aleksandrawach4@gmail.com

Received: 15.02.2021 Accepted: 21.06.2021 Early publication date: 11.08.2021

This article is available in open access under Creative Common Attribution-Non-Commercial-No Derivatives 4.0 International (CC BY-NC-ND 4.0) license, allowing to download articles and share them with others as long as they credit the authors and the publisher, but without permission to change them in any way or use them commercially. 
these important clinical problems in patients with CMBs are not yet fully understood, which means that the published recommendations are mainly based on observational studies or on the experience of experts [5-9].

This review summarises today's evidence-based clinical data regarding $\mathrm{CMBs}$ and their impact on IS and ICH risk.

\section{State of the art}

\section{Definition and prevalence}

Signs of small vessel disease (SVD) on conventional MRI include recent small subcortical infarcts, white matter magnetic resonance (MR) hyperintensities, lacunes, prominent perivascular spaces, cerebral microbleeds, and atrophy [10]. The term ' $\mathrm{CMB}$ ' is in fact a radiological concept that aims to illustrate microscopic pathology of perivascular hemosiderin deposits corresponding to small foci of past bleeding $[10,11]$. Support for this notion has been provided in post mortem correlative MR and histopathological studies. Histopathologically, areas of signal loss on gradient echo $\mathrm{T} 2{ }^{*}$-weighted sequences in the brains of deceased patients represent hemosiderin deposits indicating previous extravasation of blood [Supplementary reference 1,2]. CMBs develop alongside small arteries, arterioles or capillaries, usually demonstrating fibrolipohyalinosis or amyloid microangiopathy. Therefore, hemosiderin-laden macrophages are presented in their proximity [Supplementary reference 3]. In the population-based Mayo Clinic Study of Ageing, the age- and sex-specific prevalence of core cerebrovascular disease lesions (infarctions, cerebral microbleeds, and white-matter hyperintensities detected with magnetic resonance imaging) were assessed. Among 1,462 participants without dementia, core cerebrovascular disease features increased with age. The prevalence of CMBs was $13.6 \%$, of infarcts $11.7 \%$, and of abnormal white-matter hyperintensities $10.7 \%$. Infarcts and cerebral microbleeds are more common among men. In contrast, abnormal white-matter hyperintensities are more common among women aged 60 to 79 and men aged $80+[10]$.

Hemosiderin deposits are superparamagnetic, and thus they show considerable internal magnetisation and magnetic susceptibility into the MRI magnetic field [10]. Their detection on MRI demands proper selection of appropriate sequences. $\mathrm{CMBs}$ are not visible in computed tomography. Consequently, the sensitivity of CMB detection varies with the MRI parameter i.e. pulse sequence, spatial resolution, magnetic field strength, and post-processing method [11]. It is estimated that CMBs occur in 3-7\% of healthy people aged 45 to 50 . Their presence increases with age: in people $80+$, the prevalence of CMBs varies from $17.8 \%$ to $38.3 \%$ [ 1 , 2 ] and is higher in men [Supplementary reference 3,4]. In the Mayo Clinic Study of Ageing, CMB frequency increased with age with each succeeding decade (11\% aged 60-69, 22\% aged $70-79$, and $39 \%$ aged $80+$ ) [Supplementary reference
5]. A higher presence of CMBs in also reported in patients with first-ever and recurrent haemorrhagic or ischaemic stroke, Alzheimer's Disease, vascular cognitive impairment or vascular dementia, hypertensive vasculopathy and cerebral amyloid angiopathy (CAA) [2, 12-15]. Apart from dementia, the presence of multiple CMBs is also associated with a global neuropsychiatric disorder burden, in particular symptoms of depression and disinhibition [Supplementary reference 6]. A high extent of CMBs may also induce parkinsonism and other motor symptoms such as gait disturbances. CMBs occur more commonly in PD patients with dementia than in those without dementia [Supplementary reference 7, 8].

\section{Risk factors for CMBs}

CMBs can be associated with some of the classical cerebrovascular risk factors, including male gender, advancing age, arterial hypertension $(\mathrm{AH})$, and cigarette smoking $[3,16,17]$. However, any association between CMBs and diabetes [18] and dyslipidemia [19] has been inconsistent across published reports. In a population of patients from the Rotterdam Scan Study, the prevalence of CMBs gradually increased with age, from $6.5 \%$ in persons aged 45 to 50 to $35.7 \%$ in participants aged $80+$ [2].

The number of CMBs is positively correlated with blood pressure values, and $\mathrm{CMBs}$ can be interpreted as a type of target organ damage due to chronic hypertension [20]. The results of a study on a general population in Sweden showed that both lobar and non-lobar CMBs were associated with the presence of $\mathrm{AH}$ [17]. Among cardiovascular risk factors determining the presence of CMBs in the lobar location, amyloid angiopathy and increased diastolic blood pressure have been identified [2]. A correlation between the duration of atrial fibrillation $(\mathrm{AF})$ and the progression of CMBs during the observation period has been also reported [21].

Alcohol overuse may induce CMBs. In the AGES-Reykjavik study, heavy alcohol consumption increased the presence of CMBs in deep structures when compared to light or moderate consumption [1]. CMBs also constitute a ubiquitous manifestation of traumatic brain injury of all severities, and their presence is strongly associated with that of traumatic axonal injury (TBI). About $40 \%$ of the patients who died during the acute phase of TBI, and $47 \%$ of those who survived 12 months after a TBI, showed multifocal, perivascular and parenchymal CMBs in the grey matter [22].

CMBs develop in a high percentage of patients with brain tumours treated with radiation therapy within the first years after treatment. Significant factors for the development of a $\mathrm{CMB}$ include younger age at time of proton beam radiation therapy (PBT), a higher maximum radiation therapy dose, and a higher percentage and a higher volume of brain exposed to $\geq 30$ Gy [23]. Bacterial endocarditis is also a condition often associated with the presence of CMBs [24]. A negative impact of obesity, and a positive impact of fish oil consumption, on the occurrence of CMBs have been observed [25]. The mere presence of a CMB is also a risk factor for its further progression $[2,26]$. 


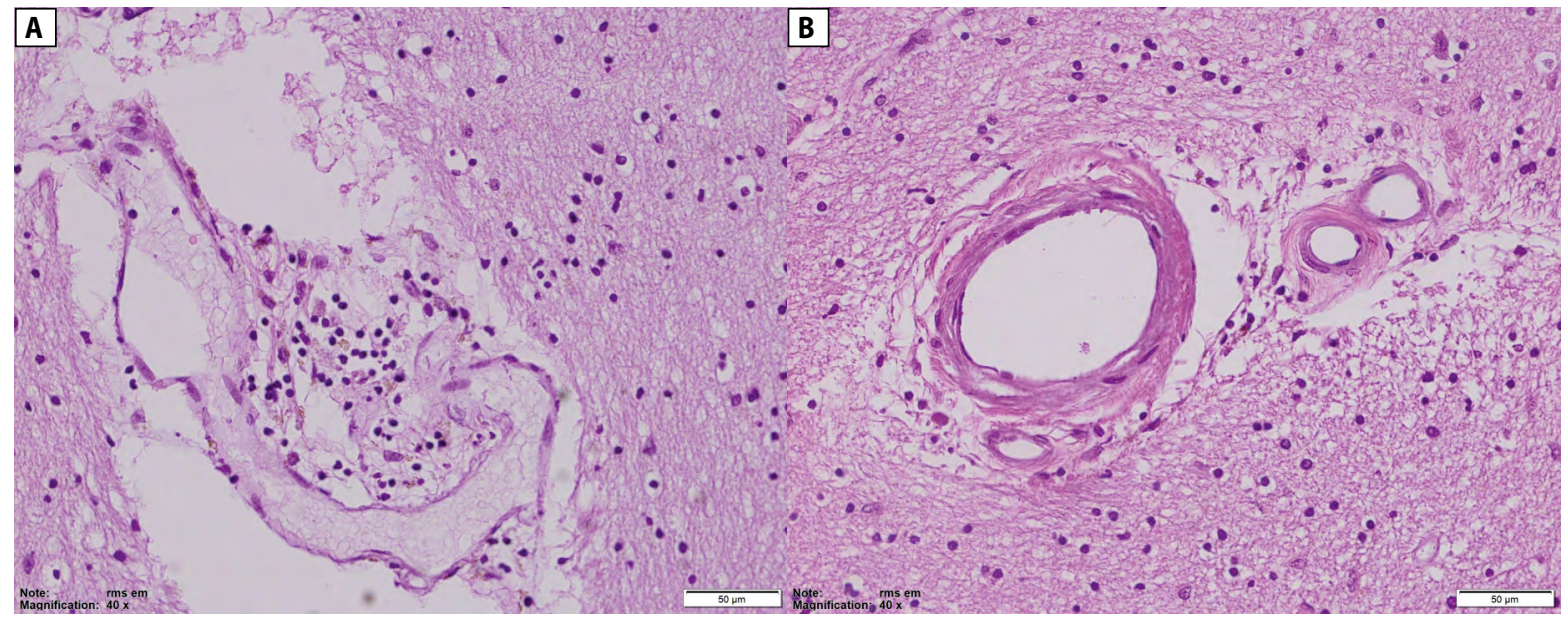

Figure 1. A. Perivenular space widening with accumulation of hemosiderin-laden macrophages and lymphocytes (HE, 400x) (Department of Pathology and Neuropathology, Medical University of Gdansk); B. Cerebral arterioles of different diameters presenting sclerotic changes and early perivascular space widening with single macrophages (HE, 400x) (Department of Pathology and Neuropathology, Medical University of Gdansk)

Neuropathology

Neuropathologically, SVD has been referred to using a wide range of terms, such as status fibrosus, status lacunaris or entity of hypertensive Binswanger's encephalopathy [27]. Age, vascular risk factors, and genetic predispositions are connected to neuro-vascular unit and cerebral small blood vessel (diameter 30-400 um) wall alterations. There are rare inherited forms and, sporadic types prevalent in older patients, with the most common being hypertensive arteriopathy (deep perforator arteriopathy) and cerebral amyloid arteriopathy (CAA).

Pathological changes of blood vessels in hypertensive arteriopathy include arteriosclerosis, fibrinoid necrosis and, although this is a term now less commonly used, lypohyalinosis. These changes are caused initially by endothelial and blood-brain barrier dysfunction. The molecular pathogenesis of arteriolosclerosis is not well understood, but its main steps encompass structural changes of the basement membranes, progressive loss of smooth myocytes, intimal thickening, replacement by collagen fibres (fibrosis), and protein depositions.

A second form, sporadic CAA, can occur in or outside an $\mathrm{AD}$ setting, and is characterised by blood vessel wall deposition of amyloid B protein (mainly AB40). AB in the soluble form is eliminated from the brain within the interstitial fluid along the vessels and along glial water channels of the glymphatic system. CAA intensity and presence have been shown to correlate with APOE specific alleles, and cerebral B-amyloid burden in PET studies.

CMBs correspond to perivascular hemosiderin-laden macrophages, hemosiderin deposits, iron-positive siderophages and small erythrocytic extravasations (Fig. 1A, B). They are identified in the setting of ICH and ischaemic stroke, and in $\mathrm{AD}$, and are more frequently seen with increasing age. Some studies show also relations to the ischaemic mechanisms. In hypertensive arteriopathy, CMBs are found in the deep grey matter, white matter, and infratentorially, while lobar (cortico-subcortical) mainly occipital or frontal lesions correspond to CAA.

The mixed type of CMB distribution points to the coexistence of both types of vascular pathology; in addition, some authors have proposed that these two diseases create a continuum of age-related vascular pathology [28]. Some studies have shown no neuropathological evidence for a topographical relation between CMB and CAA [29]. However, there have been few radiological-neuropathological correlation studies in CMB . Pathophysiologically, the causes and consequences of CMB are multifactorial: impaired vasodilatation and autoregulation, loss of elasticity, vessel stiffening, aberrant blood flow, interstitial fluid drainage, fluctuation in blood flow, hypoperfusion, inflammation (microglial activation), myelin dysfunction, and finally neurodegeneration [30, 31].

\section{Genetics}

Genetic factors determining the presence of CMBs include polymorphisms linked with sporadic CMBs and less common mutations seen with familial conditions. The most common gene polymorphism associated with sporadic $\mathrm{CMBs}$ is the apolipoprotein $\mathrm{E}$ (APOE) gene on chromosome 19. Apolipoprotein $\mathrm{E}(\mathrm{ApoE})$ is genetically associated with cerebral $\beta$-amyloidosis $(A \beta)$. ApoE has a determining role in the progression of $A \beta$ deposition, since having the $\mathrm{APOE}$ $\varepsilon 2$ and $A P O E \varepsilon 4$ alleles is the major risk factor for CAA and Alzheimer's Disease (AD). APOE $\varepsilon 2$ and APOE $\varepsilon 4$ have each been independently associated with lobar CMBs [31]. Essential hypertension is a disease with a complex and multifactorial aetiology inherited by poligenes. Allelic variants, so-called 'candidate genes', only predispose to higher pressure values, 


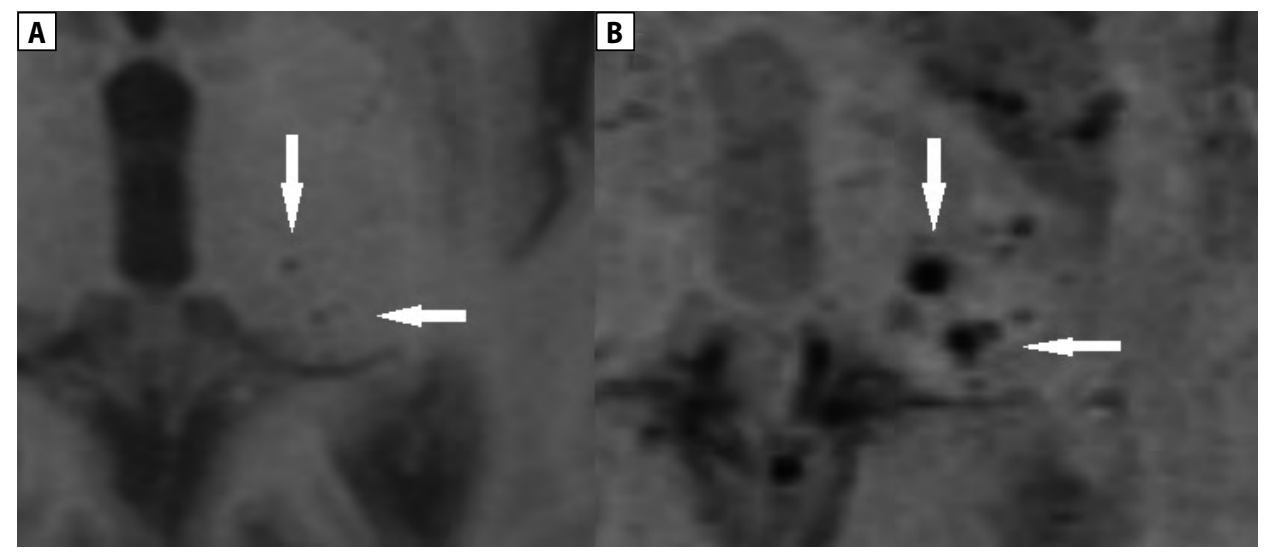

Figure 2. 'Blooming effect' in T1 (A) and T2* GRE (B) images (Department of Radiology of Holy Spirit Specialist Hospital in Sandomierz)

and only their combined action with environmental factors leads to an increase in blood pressure [32].

\section{MRI diagnostics}

The basis of blood-breakdown product detection in patients with CMBs is sequence T2*-GRE. MRI images in this sequence are typically larger than the physical size of the underlying hemosiderin deposits. This phenomenon is called the 'blooming effect' and significantly aids visual interpretation of CMBs [Fig. 2A, B]. The extent of blooming varies according to the MRI sequence parameter, especially echo, but also magnetic field strength $(7 \mathrm{~T}>3 \mathrm{~T}>1,5 \mathrm{~T}$ ), slice thickness, flip angle, spatial resolution (3D $>2 \mathrm{D})$ and image postprocessing technique (susceptibility-weighted imaging, SWI) $[10,15,34]$. SWI is another particularly effective diagnostic technique in the detection of CMBs (Fig 2A, B) [3, 34]. Studies have shown that SWI can detect significantly more CMBs (at least 67\% more) compared to conventional T2*-GRE [35]. CMBs are characterised by a lack of signal hyperintensity in the $\mathrm{T} 1$ and T2 sequences.

According to the current consensus, $\mathrm{CMBs}$ are defined as: hypointense lesions (black) on T2*-GRE MRI, round or ovoid, well defined, small, not seen well on T1- or T2-weighted MRI, with the necessary exclusion of clinical history of traumatic diffuse axonal injury, and where at least half of the lesion is surrounded by brain parenchyma $[15,34]$.

\section{Differential diagnosis}

CMBs are by definition smaller than $5-10 \mathrm{~mm}$ in diameter. CMBs should be distinguished from mimics and artifacts. Various structures in the brain can give small, dot-like, low-signal areas on T2*-GRE or SWI MRI e.g. blood vessels in the subarachnoid space, calcifications of the basal ganglia, cavernous malformations, haemorrhagic micrometastases especially from melanoma or renal cell carcinoma, and post-traumatic changes. Hence, the diagnostician must carefully review contiguous scans using different MRI sequences and sometimes compare the suspected areas using CT scans $[15,36]$.

\section{Distribution on MRI}

Yakushiji et al. analysed data from 8,595 stroke-free individual participants aged between 55 and 75 from 11 studies for the presence and distribution of CMBs. The authors compared eastern (East Asian) and western (Caucasian) populations. They found that Eastern and Western general populations have different anatomical distributions of CMBs. In their analysis, Eastern populations had higher odds of deep and/ or infratentorial or mixed CMBs [37]. CMBs in the lobar and deep locations are associated with hypertensive arteriopathy [20] [Supplementary figure 1a]. Age and APOE4 carrier status act through amyloid load to increase the risk of lobar CMBs, especially located in the occipital and parietal regions of the brain [38], although in patients with amyloid angiopathy CMBs have also been observed located infratentorially [39] [Supplementary figure 1b]. Mixed-location CMBs have been found to be a biomarker of neurodegeneration in a memory clinic population [40]. Also, the Framingham population study showed that hypertension increased the risk of any $\mathrm{CMB}$ and, in deep and mixed locations, low total cholesterol and APOE $\varepsilon 4$ increased the risk of lobar $\mathrm{CMB}$, and that statin use increased the risk of lobar and mixed location CMB [Supplementary reference 4]. Different distributions of CMBs have been observed in patients with Parkinson's Disease [4], although some studies have indicated an occurrence of strict lobar CMBs in patients with non-dementia Parkinson's Disease [41].

In order to assess the location of, and increase in, the number of CMBs, scales called 'MARS' (Microbleed Anatomical Rating Scale, Tab. 2, Supplementary reference 1) and 'BOMBS' (Brain Observer Microbleed Scale, Tab. 2, Supplementary reference 2) have been created with the possibility of mapping brain structures.

\section{Clinical implications}

Risk of ischaemic and haemorrhagic stroke

In patients with ischaemic stroke (IS), the presence of CMBs is associated with advanced age, diabetes and the previous use of antithrombotics. CMBs located in deep structures 
are also associated with the presence of arterial hypertension $[18,42]$. An increased risk of ischaemic and haemorrhagic stroke itself has been also reported in patients with CMBs $[21,43,44]$. In a meta-analysis of 13,864 patients from five population-based studies, CMB presence was significantly associated with the incidence of IS and ICH. Pooled analysis of 7,672 patients with ischaemic stroke/TIA (CMBs vs.no CMBs) from 19 studies showed that CMB was associated with an increased risk of recurrent IS and a crude risk of ICH during follow-up [36]. Patients with IS or TIA with CMBs are three times more likely to have a subsequent ICH $[21,45]$ or recurrent IS [45]. The predictors of ICH in AIS are age, high NIHSS score, and deep, lobar and cortico-subcortical distribution of CMBs. The risk and mortality of ICH increase with the quantity of CMBs $[21,46]$.

An observational prospective study based on $168 \mathrm{ICH}$ survivors who underwent 1.5T MRI at ICH onset (median follow up 3.4 years) showed that prognostic and associated factors of incident CMBs differed according to the index ICH location. Whereas in lobar ICH, incident CMBs were associated with haemorrhagic biomarkers, in non-lobar ICH the ischaemic burden increased [47]. In the MISTRAL study (MIcrobleeds predict STRoke in ALzheimer's) carried out in 333 patients with $\mathrm{AD}$ (in one in three of whom a $\mathrm{CMB}$ was imaged), the main measures were stroke-related mortality, incident stroke, and ICH. Patients with AD with lobar CMBs had an increased risk of stroke and stroke-related mortality, and the presence of non-lobar CMBs was associated with an increased risk of cardiovascular events and cardiovascular mortality [48].

\section{Dementia and neurodegenerative diseases}

No strong association between the presence of CMBs and the development of dementia has been shown in prospective studies. However, adjusted meta-analysis of three population-based studies (Rotterdam, Framingham Heart and AGES Reykjavik), which included dementia-free participants at baseline, revealed that CMBs were independently associated with a marginally increased risk of all-cause incidence of dementia $[5,49]$. In studies on an elderly population, CMB presence at baseline was associated with a doubled risk of dementia in the crude meta-analysis, although this was not confirmed in the adjusted meta-analysis $[50,51]$.

In a Japanese observational study, the presence of CMBs in patients with dementia was not associated with deterioration of memory function. But the presence of more than one CMB, and their mixed location, affected the development of dementia regardless of its clinical picture [52]. A report concerning stroke clinic patients showed that CMBs were consistently associated with frontal-executive impairment and had prognostic relevance for long-term cognitive outcome [53].

CMBs are associated with decreased cerebrospinal fluid amyloid levels and are related to the ApoE e4 allele, as well as other imaging manifestations typical of small vessel disease [Supplementary reference 9]. CMBs are found in c.24-33\% of Alzheimer's Disease dementia patients [Supplementary reference 10-12]. In patients with $\mathrm{AD}$ or vascular dementia, those with lobar-only CMBs have a higher amyloid burden than those with mixed lobar and deep CMBs or deep-only CMBs. Apolipoprotein E $\varepsilon 4$ homozygosity is associated with a greater risk of lobar CMBs [Supplementary reference 13].

The mixed location of CMBs is thought to be associated with neurodegenerative diseases of the nervous system [54]. CMBs are often detected in Parkinson's Disease, but different localisations of CMBs, including deep brain hemispheres, are more often given in this disease [4]. Kim et al. compared the occurrence of CMBs in patients with PD and multiple system atrophy (MSA), and found no difference between the number and distribution of CMBs in both groups of patients [55]. No reports of CMBs in other neurodegenerative diseases have been found.

\section{Chronic Kidney Disease and haemodialysis}

Chronic kidney disease is mentioned as one of the risk factors for CMBs, especially in patients treated with haemodialysis. A decrease in eGFR (estimated glomerular filtration rate) is associated with the occurrence of CMBs and an increase in their number [56]. MRI studies of cohorts of dialysed patients have shown asymptomatic markers of small vessel disease, including silent cerebral infarction, white matter hypersensitivity, and CMBs. However, all studies evaluating the problem of CMBs in dialysis patients to date have been conducted in Asian populations. The presence of these changes was associated with the future occurrence of strokes, memory impairment, and dementia [57]. Among 180 patients examined by Qian et al., 36.1\% had detected CMBs. Deeply located CMBs were significantly associated with haemodialysis treatment, mean arterial pressure (MAP) and the number of lacunar infarctions, but were not associated with dialysis modality or heparin use [58].

\section{Stroke course and treatment}

\section{Global risk and outcome}

Sakuta et al. found that the presence of CMBs is predicts poor outcome in minor ischaemic stroke patients [59]. Among 1,963 participants of the Framingham Heart Study, with an average follow-up of 7+ years, CMBs were not strongly associated with increased mortality of any cause, and after adjusting for cardiovascular risk factors and preventative medication, there was no statistical significance [60]. In the Rotterdam Study, 3,979 participants were observed for $5+$ years. Localisation of CMBs in deep structures of the brain and subtentorial areas was significantly associated with an increased risk of mortality of any cause, regardless of cardiovascular risk factors. The risk of mortality increased with the number of CMBs [61].

The results of the PROspective Study of Provastatin in the Elderly at Risk (PROSPER) showed that in the 7-years follow up, the presence of CMBs was associated with a six-times 
greater risk of stroke-related death. Individuals with lobar CMBs had a seven-fold increase in stroke-related deaths (but not cardiovascular deaths), and individuals with non-lobar CMBs had a doubled risk of cardiovascular (but not stroke-related) death [62].

Data from the prospective study by Soo et al. showed that the risk of increase in mortality from ICH with quantity of CMBs was as follows: 0.6\% (no CMBs); 0.9\% (1 CMB); 1.5\% (2-4 CMBs); and 3.8\% (5 or more CMBs). Mortality from IS and myocardial infarction did not increase with quantity of CMBs [46].

In an overall meta-analysis including studies across different populations (IS/TIA; memory clinic high risk elderly cohort and patients from population-based studies) of 14,433 participants, CMB presence was an independent predictor of all-cause mortality [46]. In the MISTRAL study, in a cohort of patients with Alzheimer's Disease, lobar CMBs increased the risk of fatal stroke by more than 30 times, and CMBs located in deep structures of the brain led to a 12-fold increased risk of cardiovascular death [48].

\section{Outcome after reperfusion strategies}

Due to the destruction of the walls of the small arteries and arterioles adjacent to CMBs by lipohyalinosis, formed microaneurysms, hypertension and/or cerebral amyloid angiopathy, there is a predisposition towards brain haemorrhage. The risk of intracerebral haemorrhage increases if the patient is undergoing reperfusion therapy or is being treated with anticoagulants.

Therefore, multiple CMBs should serve as an especially loud warning of a potential risk for major brain bleeding when thrombolytics and antithrombotic agents are being considered [Supplementary reference 14].

\section{IV-thrombolysis}

Intravenous thrombolysis with rt-PA is the most widely used treatment for AIS $[8,9]$ Clinical studies and meta-analyses evaluating the association of CMBs with outcomes of AIS patients treated with IVT have shown contradictory results [63-70]. Some of these studies did not indicate a relationship between high CMB burden and poor long-term outcome [66, 67], but in contrast other studies did point to such an association [68-70]. A multicentre prospective European study and a meta-analysis by Arca et al. showed that only a high number of CMBs $(\geq 10)$ was associated with higher mortality in patients treated with intravenous thrombolysis (IVT) [63]. Similar conclusions resulted from the multistep algorithm to model 90-day modified Rankin Scale scores in patients with $\leq 10$ vs. $>10$ CMBs who did or did not receive IVT developed by Schlemm et al. [64]. In the meta-analysis by Wang et al., no effect of CMBs on mortality in IVT patients was found [65].

The results of studies in the context of occurrence of sICH after IVT in patients with CMBs detected before treatment are also contradictory $[71,72]$. Zand et al. indicated that a high $\mathrm{CMB}$ burden $(>10)$ is associated with a higher risk of sICH [73]. The same conclusion was confirmed by other studies and meta-analyses $[65,73]$. The recent meta-analysis by Yan et al. of 2,407 participants from nine studies showed that pretreatment CMBs were associated with increased incidence of sICH in AIS patients receiving IVT. However, these results were not convincing enough to establish the presence of a $\mathrm{CMB}$ as a contraindication to IVT [74].

\section{Endovascular thrombectomy (EVT)}

Prospective data regarding the impact of CMBs on the safety and efficacy of mechanical thrombectomy in patients with ASI remains limited. Choi and al. analysed the impact of CMBs on long-term outcome following recanalisation in patients with AIS. They found that the presence of a $\mathrm{CMB}$, and high burden and lobar location, are independent predictors of poor outcomes, and may increase sICH especially in patients with recanalisation after large vessel occlusion, more than in those without recanalisation [75]. In the study by Shi et al., $6.8 \%$ had $\geq 2 \mathrm{CMBs}$, and only 1 patient had $\geq 5 \mathrm{CMBs}$. The authors showed that the presence of a CMB did not increase haemorrhagic transformation (HT) and mortality following EVT for AIS [76].

To date, only one meta-analysis based on the results of four studies with a total of 598 patients has been published. CMBs were present in $18 \%$, and $\geq 5$ CMBs in only $1 \%$ of patients. The risk of ICH after EVT did not significantly differ between patients with and without CMBs [77].

\section{Carotid endarterectomy (CEA) and angioplasty with stenting (CAS)}

Only two reports have discussed the problematic presence of CMBs in patients undergoing CAS. Among the whole group of patients treated by Kakumato et al., 20.5\% had CMBs initially, and $8 \%$ developed new CMBs straight after CAS. New CMBs appeared on the same side of CAS in all patients. New CMBs appeared significantly more frequently in the $\mathrm{CMB}$-positive group than in the CMB-negative one [78]. This observation confirmed the results of the study conducted by Ito et al., which found that new CMBs also developed after CAS, mostly in the territory of the treated carotid artery [79].

In a multicentre European study, 162 patients were treated with CEA or CAS. In both groups, there was no manifestation of ICH after surgery. CMBs appeared in only $6.0 \%$ of patients after CAS, and in $6.4 \%$ after CEA, without statistical significance between the groups [80].

\section{Antithrombotic therapies}

\section{Antiplatelets}

There has been long-running uncertainty as to whether during chronic use of antiplatelet agents there is incidence of $\mathrm{CMBs}$ or an increase in the number of them, and what their location is, and whether IS and/or ICH occurs more frequently. 
Many studies have been carried out on this subject, but they have mainly been observational studies.

There have been some systemic reviews and meta-analyses discussing the relationship between antiplatelet drug use and CMBs [81-83]. The meta-analysis by Liu et al., based on 11 studies involving 10,429 participants, revealed a significant relationship between antiplatelet therapy and the occurrence of CMBs in both ICH and IS patients. In the case of stratification based on ethnicity, the relationship between antiplatelet therapy and CMBs was at a similar level of significance for ICH and IS for an Asian population, but was not significant for ICH and IS for patients from European countries [81]. A review of the literature including 1,460 patients with ICH and 3,817 with IS/TIA showed that CMBs were almost three times more common in the group of $\mathrm{ICH}$, and almost six times more often seen in patients using antiplatelet drugs [82]. The meta-analysis conducted by Wang at al., based on the results of 10 studies, showed that patients with multiple CMBs had an almost quadrupled risk of developing ICH compared to patients with a single CMB. A very strong relationship has been found between the presence of $\mathrm{CMBs}$ and the subsequent occurrence of ICH in patients treated with antiplatelet agents [83]. The meta-analysis by Qui et al. was based on the results of 37 studies with a total of 20,988 participants. The analysis showed that CMBs were more frequent in antiplatelet users, and in those in strictly lobar rather than in than deep or infratentorial locations. ICH was higher in participants with CMBs than in those without CMBs [84].

The results of a recently conducted study emphasise that the duration of antiplatelet therapy can influence the prevalence of CMBs and the incidence of ICH [85].

\section{Oral anticoagulants}

Atrial fibrillation (AF) quintuples the risk of IS. Therefore, to reduce this risk, anticoagulants are indicated [86]. For this reason, for many years vitamin $\mathrm{K}$ antagonists (VKAs) have been used. We have had the results of several studies assessing the risk of ICH in patients using VKAs, although most of these studies did not take into account the presence of CMBs. NOACs (novel oral anticoagulants) have been used for several years, but the issue of any correlation between the occurrence of a CMB and the safety of treatment has not been properly studied.

Previous studies, not assessing the presence of CMBs in the MRI, showed that NOAC-related ICH patients had lower baseline haematoma volume and were less likely to have severe neurological deficits ( $>10$ points according to NIHSS-National Institutes of Health Stroke Scale score) on admission than VKA-ICH patients [87].

In turn, in the international collaborative multicentre pooled analysis CROMIS-2 (Clinical Relevance of Microbleeds In Stroke), no differences in baseline ICH volume, haematoma expansion, 90-day mortality, or functional outcome in ICH-patients treated with NOAC and VKA were found [88].
Graff-Radford et al. showed that anticoagulant use correlated with the presence of CMBs in the general population, and that the predictors for presence/absence of CMBs included older age at magnetic resonance imaging and male sex. The predictors of CMB count in the CMB-positive group were male sex and amyloid load detected with positron emission tomography (PET) [Supplementary reference 15].

In the multicentre prospective, observational study RASUNOA (Rationale and Design of the Registry of Acute Stroke Under Novel Oral Anticoagulants) location and counts of CMBs in patients with IS and ICH prior treated with NOAC were analysed. The proportion of patients with at least one $\mathrm{CMB}$, and the absolute number of CMBs, were higher in the ICH group [89]. Lioutas et al. evaluated the incidence of CMBs in ICH patients treated with anticoagulants before stroke onset. In the study group, CMB prevalence was 51\% (52\% in VKA, $48 \%$ in NOAC). NOAC patients had a lower CMB count, and $\geq 5$ CMBs were less prevalent in the NOAC group [90].

Balancing the risks of recurrent ischaemic stroke and intracranial haemorrhage is important for patients treated with antithrombotic therapy after ischaemic stroke or transient ischaemic attack.

In the aforementioned CROMIS-2 study of patients with atrial fibrillation anticoagulated after recent IS or TIA, CMBs were independently associated with sICH risk [91]. The results of the meta-analysis by Charidimou et al. based on a group of 1,552 patients pointed to a particular risk of ICH in patients in whom $\geq 5$ CMBs had been detected [92].

In the pooled analysis of individual patient data from the Microbleeds International Collaborative Network (MICON), which includes 38 hospital-based prospective cohort studies from 18 countries, the authors found the novel MICON-intracranial haemorrhage (MICON-ICH) and MICON-ischaemic stroke (MICON-IS) risk scores - which include clinical variables and MRI-detected cerebral microbleeds - to predict intracranial haemorrhage in patients taking antithrombotic therapy after ischaemic stroke or transient ischaemic attack. These scores are new means by which to assess the longterm risk of intracranial haemorrhage and ischaemic stroke [Supplementary reference 16]. In Greenberg's opinion, the MICON-ICH risk score is substantially better than the discrimination offered by scales such as HASBLED that do not incorporate CMBs [Supplementary reference 17].

\section{Statins}

The results of studies assessing the impact of lipid levels and the effect of drugs used to regulate their level are inconsistent $[19,93]$. Some of them have indicated that low serum cholesterol level and/or triglyceride level is associated with an increased risk of ICH. A meta-analysis based on 43 studies with a combined total of 317,291 patient-years of follow-up indicated that, in patients with ICH, statins did not increase recurrent ICH. In survivors of IS, although statins substantially and significantly reduced IS, there was a non-significant increase 
Table 1. Cerebral microbleeds in the guidelines of expert groups and scientific societies

American Heart Association/ American Stroke Association: Scientific rationale for inclusion and exclusion criteria for intravenous alteplase in acute ischaemic stroke [97]

American Heart Association/ American Stroke Association: Prevention of stroke in patients with silent cerebrovascular disease [98]

American Heart Association/ American Stroke Association: 2018 guidelines for early management of patients with acute ischaemic stroke [9]

European Stroke Organisation - Karolinska Stroke Update Conference: Consensus statements and recommendations from ESO Karolinska Stroke Update Conference [99]

European Stroke Organisation (ESO) guidelines on intravenous thrombolysis for acute ischaemic stroke [Supplementary reference 23]

Decision algorithms for direct oral anticoagulant use in patients with nonvalvular atrial fibrillation: a practical guide for neurologists [100]

European Society of Cardiology (ESC) guidelines for management of atrial fibrillation developed in collaboration with EACTS [8]
Intravenous alteplase has not been shown to increase sICH rates in patients with CMBs. Intravenous alteplase administration in these patients is therefore reasonable (Class lla; benefit $>$ risk)

We suggest that, for patients with nonvalvular atrial fibrillation in whom anticoagulation is indicated but who are considered at particularly high risk of future ICH on basis of microbleed number and location, it may be reasonable to administer dabigatran, rivaroxaban, apixaban, or edoxaban in preference to warfarin. Another alternative to warfarin anticoagulation that might be considered is percutaneous closure of left atrial appendage

In eligible patients who have previously had a small number (10 or fewer) of CMBs demonstrated on MRI, administration of IV alteplase is reasonable (Class Ila; benefit > risk); In eligible patients who have previously had a high burden of CMBs (more than 10) demonstrated on MRI, treatment with IV alteplase may be associated with an increased risk of $\mathrm{sICH}$, and benefits of treatment are uncertain (Class Ilb; benefit $\geq$ risk)

Routine MRI assessment of small vessel disease including CMBs is not recommended (Grade C; benefits outweigh risk); Oral anticoagulants in patients with evidence of CMBs should not be withheld (Grade C); NOACs should preferentially be used over VKA in NVAF (Grade C)

For patients with acute ischaemic stroke of $<4.5 \mathrm{~h}$ duration, for whom cerebral microbleed burden is unknown or is known to be low $(<10)$, we suggest intravenous thrombolysis with alteplase. Quality of evidence: Low; Strength of recommendation: Weak For patients with acute ischaemic stroke of $<4.5 \mathrm{~h}$ duration, for whom cerebral microbleed burden has been previously reported to be high ( $>10$ ), we suggest no intravenous thrombolysis. Quality of evidence: Low; Strength of recommendation: Weak

In patients after $\mathrm{ICH}$, when anticoagulation is contraindicated (among other things on detection of $>5$ cortical CMBs), surgical ablation or percutaneous left atrial appendage closure should be considered

Among factors supporting lack of return to anticoagulation in patients with atrial fibrillation after $\mathrm{ICH}$, numerous (> 10) CMBs are listed of ICH. Nonetheless, statins show clear benefits in reducing mortality and improving functional outcome, irrespective of stroke subtype [94]. The conclusion of the Rotterdam Study was that low serum triglyceride level was associated with the presence of deep or infratentorial CMBs [95].

A recent review based on seven studies of 3,671 participants presented the hypothesis that statins treatment is not associated with CMBs overall, but may increase the risk of lobar CMBs formation [96].

\section{Current guidelines}

Nowadays, reperfusion therapy is widely used in AIS patients, and stroke prevention relies on chronic anticoagulation with a tendency towards aggressively initiating treatment. Unfortunately, the eligibility criteria for reperfusion procedures and the scales currently used to qualify for secondary stroke prevention, do not take the presence of CMBs into account [Supplementary reference 18-24].

All recommendations regarding the use of antiplatelet, anticoagulant or IVT and EVT in patients with CMBs are experts' opinions mostly based on the results of observational studies or on randomised clinical trials with relatively small groups of patients. However, despite omitting the issue of $\mathrm{CMBs}$, they constitute a guideline for managing patients in various clinical cases.
In 2013, Fisher proposed MRI screening for chronic anticoagulation in AF [Supplementary reference 25]. The algorithm developed by Fisher recommends MRI screening in patients aged $60+$. Among those patients who have CMBs demonstrable on MRI, a distinction is made between cortical vs. subcortical CMBs, and between findings of five or more subcortical vs. less than five subcortical CMBs. According to this algorithm, treatment should be considered in patients with cortical CMBs or at least five subcortical CMBs [97].

Following the current AHA/ASA Guidelines, no RCT of IVT and EVT in AIS with baseline MRI to identify CMBs have been conducted, so no determination of effect of baseline CMBs on the treatment safety and efficacy of alteplase and EVT with CMBs is available. In the absence of any evidence that IVT and EVT provide no benefit or cause harm in eligible patients with CMBs, withholding treatment on the basis of the presence of CMBs could lead to the exclusion of patients who would benefit from it.

The AHA/ASA also recommend the use of antiplatelet drugs and oral anticoagulants in the prevention of cardiovascular events in patients with CMBs $[8,97,98]$. The problem of CMBs in the European Stroke Organisation (ESO) [99, Supplementary reference 26] and European Association of Cardiology (ESC) [7] documents and in the Practical Guide for Neurologists published in 2019 by Canavero et al. [100] is under discussion. The current recommendations are set out in Table 1. 
Therefore, future studies should determine the number of CMBs above which we should not perform reperfusion therapy and not use anticoagulant therapy, and also answer the questions as to whether the number is the same for different $\mathrm{CMB}$ locations and whether the presence of other cardiovascular risk factors could correct this number. It is also important to identify those groups of patients that should be scanned by MRI before making therapeutic decisions.

\section{Conflict of interest: None.}

\section{References}

1. Ding J, Sigurdsson S, Garcia M, et al. Risk factors associated with incident cerebral microbleeds according to location in older people: the age, gene/environment susceptibility (AGES)-Reykjavik study. JAMA Neurol. 2015; 72(6): 682-688, doi: 10.1001/jamaneurol.2015.0174, indexed in Pubmed: 25867544.

2. Poels MMF, Vernooij MW, Ikram MA, et al. Prevalence and risk factors of cerebral microbleeds: an update of the Rotterdam scan study. Stroke. 2010; 41(10 Suppl): S103-S106, doi: 10.1161/STROKEAHA.110.595181, indexed in Pubmed: 20876479.

3. Kakar P, Charidimou A, Werring DJ. Cerebral microbleeds: a new dilemma in stroke medicine. JRSM Cardiovasc Dis. 2012; 1(8): 2048004012474754, doi: $10.1177 / 2048004012474754$, indexed in Pubmed: 24175079.

4. Yamashiro $\mathrm{K}$, Tanaka $\mathrm{R}$, Shimo $\mathrm{Y}$, et al. The prevalence and risk factors of cerebral microbleeds in patients with Parkinson's disease. Parkinsonism Relat Disord. 2015; 21(9): 1076-1081, doi: 10.1016/j. parkreldis.2015.06.019, indexed in Pubmed: 26142208.

5. Charidimou A, Shoamanesh A, Al-Shahi Salman R, et al. Cerebral amyloid angiopathy, cerebral microbleeds and implications for anticoagulation decisions: The need for a balanced approach. Int J Stroke. 2018; 13(2): 117-120, doi: 10.1177/1747493017741384, indexed in Pubmed: 29125055.

6. Choi KH, Kim JH, Kang KW, et al. Impact of microbleeds on outcome following recanalization in patients with acute ischemic stroke. Stroke. 2018 [Epub ahead of print]: STROKEAHA118023084, doi: 10.1161/ STROKEAHA.118.023084, indexed in Pubmed: 30580721.

7. Kirchhof $P$, Benussi $S$, Kotecha D, et al. ESC Scientific Document Group. 2016 ESC Guidelines for the management of atrial fibrillation developed in collaboration with EACTS. Eur Heart J. 2016; 37(38): 2893-2962, doi: 10.1093/eurheartj/ehw210, indexed in Pubmed: 27567408.

8. Powers W, Rabinstein A, Ackerson T, et al. 2018 guidelines for the early management of patients with acute ischemic stroke: a guideline for healthcare professionals from the American Heart Association/ American Association. Stroke. 2018; 49(3): e46-99, doi: 10.1161/ STR.0000000000000158, indexed in Pubmed: 29367334.

9. Błażejewska-Hyżorek B, Czernuszenko A, Członkowska A, et al. Wytyczne postępowania w udarze mózgu. Polski Przegląd Neurologiczny. 2019; 15(supl. A): 30-92, doi: 10.5603/PPN.2019.0001.

10. Graff-Radford J, Aakre JA, Knopman DS, et al. Prevalence and heterogeneity of cerebrovascular disease imaging lesions. Mayo Clin Proc. 2020; 95(6): 1195-1205, doi: 10.1016/j.mayocp.2020.01.028, indexed in Pubmed: 32498775.

11. Janaway BM, Simpson JE, Hoggard N, et al. MRC Cognitive Function and Ageing Neuropathology Study. Brain haemosiderin in older peo- ple: pathological evidence for an ischaemic origin of magnetic resonance imaging (MRI) microbleeds. Neuropathol Appl Neurobiol. 2014; 40(3): 258-269, doi: 10.1111/nan.12062, indexed in Pubmed: 23678850 .

12. Vernooij MW, Ikram MA, Wielopolski PA, et al. Cerebral microbleeds: accelerated 3D T2*-weighted GRE MR imaging versus conventional 2D T2*-weighted GRE MR imaging for detection. Radiology. 2008; 248(1): 272-277, doi: 10.1148/radiol.2481071158, indexed in Pubmed: 18490493.

13. Cordonnier C, van de, Sluimer JD, et al. Prevalence and severity of microbleeds in a memory clinic setting. Neurology. 2006; 66(9): 1356-1360, doi: 10.1212/01.wnl.0000210535.20297.ae, indexed in Pubmed: 16682667.

14. Fazekas F, Kleinert R, Roob G, et al. Histopathologic analysis of foci of signal loss on gradient-echo T2*-weighted MR images in patients with spontaneous intracerebral hemorrhage: evidence of microangiopathyrelated microbleeds. AJNR Am J Neuroradiol. 1999; 20(4): 637-642, indexed in Pubmed: 10319975.

15. Greenberg SM, Vernooij MW, Cordonnier C, et al. Microbleed Study Group. Cerebral microbleeds: a guide to detection and interpretation. Lancet Neurol. 2009; 8(2): 165-174, doi: 10.1016/S14744422(09)70013-4, indexed in Pubmed: 19161908.

16. Jeerakathil T, Wolf PA, Beiser A, et al. Cerebral microbleeds: prevalence and associations with cardiovascular risk factors in the Framingham Study. Stroke. 2004; 35(8): 1831-1835, doi: 10.1161/01. STR.0000131809.35202.1b, indexed in Pubmed: 15155954.

17. Elmståhl S, Ellström K, Siennicki-Lantz $\mathrm{A}$, et al. Association between cerebral microbleeds and hypertension in the Swedish general population "Good Aging in Skåne" study. J Clin Hypertens (Greenwich). 2019; 21(8): 1099-1107, doi: 10.1111/jch.13606, indexed in Pubmed: 31274244

18. Nighoghossian N, Hermier M, Adeleine P, et al. Old microbleeds are a potential risk factor for cerebral bleeding after ischemic stroke: a gradientecho T2*-weighted brain MRI study. Stroke. 2002; 33(3): 735-742, doi: 10.1161/hs0302.104615, indexed in Pubmed: 11872897.

19. Orken DN, Kenangil G, Uysal E, et al. Lack of association between cerebral microbleeds and low serum cholesterol in patients with acute intracerebral hemorrhage. Clin Neurol Neurosurg. 2010; 112(8): 668-671, doi: 10.1016/j.clineuro.2010.05.004, indexed in Pubmed: 20627554.

20. Lyu L, Shen J, Zeng C, et al. Cerebral microbleeds are associated with blood pressure levels in individuals with hypertension. Clin Exp Hypertens. 2020; 42(4): 328-334, doi: 10.1080/10641963.2019.1665673, indexed in Pubmed: 31542967.

21. Horstmann S, Möhlenbruch M, Wegele C, et al. Prevalence of atrial fibrillation and association of previous antithrombotic treatment in patients with cerebral microbleeds. Eur J Neurol. 2015; 22(10): 1355-1362.

22. Liu J, Kou Z, Tian Y. Diffuse axonal injury after traumatic cerebral microbleeds: an evaluation of imaging techniques. Neural Regen Res. 2014; 9(12): 1222-1230, doi: 10.4103/1673-5374.135330, indexed in Pubmed: 25206786.

23. Wahl M, Anwar M, Hess CP, et al. Relationship between radiation dose and microbleed formation in patients with malignant glioma. Radiat Oncol. 2017; 12(1): 126, doi: 10.1186/s13014-017-0861-5, indexed in Pubmed: 28797254.

24. Murai R, Kaji S, Kitai T, et al. The clinical significance of cerebral microbleeds in infective endocarditis patients. Semin Thorac Cardiovasc 
Surg. 2019; 31(1): 51-58, doi: 10.1053/j.semtcvs.2018.09.020, indexed in Pubmed: 30287247.

25. van Bussel BCT, Henry RMA, Schalkwijk CG, et al. Fish consumption in healthy adults is associated with decreased circulating biomarkers of endothelial dysfunction and inflammation during a 6-year follow-up. J Nutr. 2011; 141(9): 1719-1725, doi: 10.3945/jn.111.139733, indexed in Pubmed: 21753064.

26. Lee SH, Lee ST, Kim BJ, et al. Dynamic temporal change of cerebral microbleeds: long-term follow-up MRI study. PLoS One. 2011; 6(10): e25930, doi: 10.1371/journal.pone.0025930, indexed in Pubmed: 22022473.

27. Lammie GA. Pathology of small vessel stroke. Br Med Bull. 2000; 56(2): 296-306, doi: 10.1258/0007142001903229, indexed in Pubmed: 11092081.

28. Schreiber S, Wilisch-Neumann A, Schreiber F, et al. Invited Review: The spectrum of age-related small vessel diseases: potential overlap and interactions of amyloid and nonamyloid vasculopathies. Neuropathol Appl Neurobiol. 2020; 46(3): 219-239, doi: 10.1111/nan.12576, indexed in Pubmed: 31386773.

29. Kövari E, Charidimou A, Herrmann FR, et al. No neuropathological evidence for a direct topographical relation between microbleeds and cerebral amyloid angiopathy. Acta Neuropathol Commun. 2015; 3: 49, doi: 10.1186/s40478-015-0228-9, indexed in Pubmed: 26268348.

30. Kalaria RN. Neuropathological diagnosis of vascular cognitive impairment and vascular dementia with implications for Alzheimer's disease. Acta Neuropathol. 2016; 131(5): 659-685, doi: 10.1007/ s00401-016-1571-z, indexed in Pubmed: 27062261.

31. Yates PA, Villemagne VL, Ellis KA, et al. Cerebral microbleeds: a review of clinical, genetic, and neuroimaging associations. Front Neurol. 2014; 4(205), doi: 10.3389/fneur.2013.00205, indexed in Pubmed: 24432010.

32. Wise IA, Charchar FJ. Epigenetic modifications in essential hypertension. Int J Mol Sci. 2016; 17(4): 451, doi: 10.3390/ijms17040451, indexed in Pubmed: 27023534.

33. Conijn MMA, Geerlings MI, Biessels GJ, et al. Cerebral microbleeds on MR imaging: comparison between 1.5 and 7T. AJNR Am J Neuroradiol. 2011; 32(6): 1043-1049, doi: 10.3174/ajnr.A2450, indexed in Pubmed: 21546463.

34. Nandigam RNK, Viswanathan A, Delgado P, et al. MR imaging detection of cerebral microbleeds: effect of susceptibility-weighted imaging, section thickness, and field strength. AJNR Am J Neuroradiol. 2009; 30(2): 338-343, doi: 10.3174/ajnr.A1355, indexed in Pubmed: 19001544.

35. Greenberg SM, Nandigam RN, Delgado P, et al. Microbleeds versus macrobleeds: evidence for distinct entities. Stroke. 2009; 40(7): 2382-2386, doi: 10.1161/STROKEAHA.109.548974, indexed in Pubmed: 19443797.

36. Charidimou A, Shams S, Romero JR, et al. International METAMICROBLEEDS Initiative. Clinical significance of cerebral microbleeds on MRI: A comprehensive meta-analysis of risk of intracerebral hemorrhage, ischemic stroke, mortality, and dementia in cohort studies (v1). Int J Stroke. 2018; 13(5): 454-468, doi: 10.1177/1747493017751931, indexed in Pubmed: 29338604.

37. Yakushiji Y, Wilson D, Ambler G, et al. Distribution of cerebral microbleeds in the East and West: Individual participant meta-analysis. Neurology. 2019; 92(10): e1086-e1097.

38. Graff-Radford J, Lesnick T, Rabinstein AA, et al. Cerebral microbleed incidence, relationship to amyloid burden: The Mayo Clinic Study of Aging. Neurology. 2020; 94(2): e190-e199, doi: 10.1212/ WNL.0000000000008735, indexed in Pubmed: 31801832.

39. Renard D, Tatu L, Thouvenot E. Infratentorial cerebral microbleeds in patients with cerebral amyloid angiopathy. J Stroke Cerebrovasc Dis. 2018; 27(9): 2534-2537, doi: 10.1016/j.jstrokecerebrovasdis.2018.05.015, indexed in Pubmed: 29861129.

40. Gyanwali B, Shaik MA, Venketasubramanian N, et al. Mixed-location cerebral microbleeds: An imaging biomarker for cerebrovascular pathology in cognitive impairment and dementia in a memory clinic population. J Alzheimers Dis. 2019; 71(4): 1309-1320, doi: 10.3233/ JAD-190540, indexed in Pubmed: 31524167.

41. Kim KJ, Bae YJ, Kim JM, et al. The prevalence of cerebral microbleeds in non-demented Parkinson's disease patients. J Korean Med Sci. 2018; 33(46): e289, doi: 10.3346/jkms.2018.33.e289, indexed in Pubmed: 30416409.

42. Yakushiji Y, Yokota C, Yamada N, et al. Clinical characteristics by topographical distribution of brain microbleeds, with a particular emphasis on diffuse microbleeds. J Stroke Cerebrovasc Dis. 2011; 20(3): 214-221, doi: 10.1016/j.jstrokecerebrovasdis.2009.12.001, indexed in Pubmed: 20621512.

43. Lim JS, Hong KS, Kim GM, et al. Cerebral microbleeds and early recurrent stroke after transient ischemic attack: results from the Korean Transient Ischemic Attack Expression Registry. JAMA Neurol. 2015; 72(3): 301-308, doi: 10.1001/jamaneurol.2014.3958, indexed in Pubmed: 25580844.

44. Akoudad S, Portegies MLP, Koudstaal PJ, et al. Cerebral microbleeds are associated with an increased risk of stroke: the Rotterdam study. Circulation. 2015; 132(6): 509-516, doi: 10.1161/CIRCULATIONAHA.115.016261, indexed in Pubmed: 26137955.

45. Imaizumi $T$, Horita $Y$, Hashimoto $Y$, et al. Dotlike hemosiderin spots on $\mathrm{T} 2{ }^{*}$-weighted magnetic resonance imaging as a predictor of stroke recurrence: a prospective study. J Neurosurg. 2004; 101(6): 915-920, doi: 15597750, indexed in Pubmed: 10.3171/ jns.2004.101.6.0915.

46. Soo YOY, Yang SR, Lam WWM, et al. Risk vs benefit of anti-thrombotic therapy in ischaemic stroke patients with cerebral microbleeds. J Neurol. 2008; 255(11): 1679-1686, doi: 10.1007/s00415-0080967-7, indexed in Pubmed: 19156486.

47. Pasquini M, Benedictus MR, Boulouis G, et al. Incident cerebral microbleeds in a cohort of intracerebral hemorrhage. Stroke. 2016; 47(3): 689-694, doi: 10.1161/STROKEAHA.115.011843, indexed in Pubmed: 26839348.

48. Benedictus MR, Prins ND, Goos JDC, et al. Microbleeds, mortality, and stroke in Alzheimer disease: The MISTRAL study. JAMA Neurol. 2015; 72(5): 539-545, doi: 10.1001/jamaneurol.2015.14, indexed in Pubmed: 25798556.

49. Akoudad S, Wolters FJ, Viswanathan A, et al. Association of cerebral microbleeds with cognitive decline and dementia. JAMA Neurol. 2016; 73(8): 934-943, doi: 10.1001/jamaneurol.2016.1017, indexed in Pubmed: 27271785.

50. Miwa K, Tanaka M, Okazaki S, et al. Endoscopic follow-up of 3 cases with gastrointestinal tract involvement of mantle cell lymphoma. Intern Med. 2010; 49(3): 231-235, doi: 10.2169/internalmedicine.49.2766, indexed in Pubmed: 20118601.

51. van Uden IWM, van der Holst HM, Tuladhar AM, et al. White matter and hippocampal volume predict the risk of dementia in patients with cerebral small vessel disease: The RUN DMC study. J Alzheimers Dis. 2016; 49(3): 863-873, doi: 10.3233/JAD-150573, indexed in Pubmed: 26529206. 
52. Miwa K, Tanaka M, Okazaki S, et al. Endoscopic follow-up of 3 cases with gastrointestinal tract involvement of mantle cell lymphoma. Intern Med. 2010; 49(3): 231-235, doi: 10.2169/internalmedicine.49.2766, indexed in Pubmed: 20118601.

53. Gregoire SM, Smith K, Jäger HR, et al. Cerebral microbleeds and long-term cognitive outcome: longitudinal cohort study of stroke clinic patients. Cerebrovasc Dis. 2012; 33(5): 430-435, doi: 10.1159/000336237, indexed in Pubmed: 22456577.

54. Gyawali B, Shaik MA, Tan CS, et al. Mixed-location cerebral microbleeds as a biomarker of neurodegeneration in a memory clinic population. Aging (Albany NY. 2019; 11(22): 10581-10596.

55. Kim JM. The prevalence of cerebral microbleeds in patients with Parkinson's disease and multiple system atrophy [abstract]. Mov Disord. 2017;32(Suppl 2). https://www.mdsabstracts.org/abstract/ the-prevalence-of-cerebral-microbleeds-in-patients-with-parkinsons-disease-and-multiple-system-atrophy/ (15.01.2021).

56. Kim SH, Shin DW, Yun JM, et al. Correction: Kidney dysfunction and cerebral microbleeds in neurologically healthy adults. PLoS One. 2017; 12(4): e0176901, doi: 10.1371/journal.pone.0176901, indexed in Pubmed: 28448573.

57. Naganuma T, Takemoto Y, Shoji T, et al. Cerebral microbleeds predict intracerebral hemorrhage in hemodialysis patients. Stroke. 2015; 46(8): 2107-2112, doi: 10.1161/STROKEAHA.115.009324, indexed in Pubmed: 26089331.

58. Qian $\mathrm{Y}$, Zheng Ke, Wang $\mathrm{H}$, et al. Cerebral microbleeds and their influence on cognitive impairment in Dialysis patients. Brain Imaging Behav. 2021; 15(1): 85-95, doi: 10.1007/s11682-019-00235-z, indexed in Pubmed: 31898093.

59. Sakuta K, Yaguchi H, Sato T, et al. The impact of cerebral microbleeds presence on outcome following minor stroke treated with antiplatelet therapy. Front Neurol. 2020; 11: 522, doi: 10.3389/ fneur.2020.00522, indexed in Pubmed: 32612570.

60. Romero JR, Preis SR, Beiser A, et al. Cerebral microbleeds as predictors of mortality: The framingham heart study. Stroke. 2017; 48(3): 781-783, doi: 10.1161/STROKEAHA.116.015354, indexed in Pubmed: 28143923.

61. Akoudad S, Ikram MA, Koudstaal PJ, et al. Cerebral microbleeds and the risk of mortality in the general population. Eur J Epidemiol. 2013; 28(10): 815-821, doi: 10.1007/s10654-013-9854-3, indexed in Pubmed: 24072508.

62. Altmann-Schneider I, Trompet S, de Craen AJM, et al. Cerebral microbleeds are predictive of mortality in the elderly. Stroke. 2011; 42(3): 638-644, doi: 10.1161/STROKEAHA.110.595611, indexed in Pubmed: 21233474.

63. Arca KN, Demaerschalk BM, Almader-Douglas D, et al. Does high cerebral microbleed burden increase the risk of intracerebral hemorrhage after intravenous tissue plasminogen activator for acute ischemic stroke? Neurologist. 2019; 24(1): 40-43, doi: 10.1097/ NRL.0000000000000228, indexed in Pubmed: 30586035.

64. Schlemm L, Endres M, Werring DJ, et al. Benefit of intravenous thrombolysis in acute ischemic stroke patients with high cerebral microbleed burden. Stroke. 2020; 51(1): 232-239, doi: 10.1161/STROKEAHA.119.027633, indexed in Pubmed: 31739772.

65. Wang $S, L v Y$, Zheng $X$, et al. The impact of cerebral microbleeds on intracerebral hemorrhage and poor functional outcome of acute ischemic stroke patients treated with intravenous thrombolysis: a systematic review and meta-analysis. J Neurol. 2017; 264(7): 1309-1319, doi: 10.1007/s00415-016-8339-1, indexed in Pubmed: 27885484.
66. Dannenberg S, Scheitz JF, Rozanski M, et al. Number of cerebral microbleeds and risk of intracerebral hemorrhage after intravenous thrombolysis. Stroke. 2014; 45(10): 2900-2905, doi: 10.1161/ STROKEAHA.114.006448, indexed in Pubmed: 25116882.

67. Turc G, Sallem A, Moulin S, et al. Microbleed status and 3-month outcome after intravenous thrombolysis in 717 patients with acute ischemic stroke. Stroke. 2015; 46(9): 2458-2463, doi: 10.1161/ STROKEAHA.115.009290, indexed in Pubmed: 26230857.

68. Yan S, Jin X, Zhang X, et al. Extensive cerebral microbleeds predict parenchymal haemorrhage and poor outcome after intravenous thrombolysis. J Neurol Neurosurg Psychiatry. 2015; 86(11): $1267-$ 1272, doi: 10.1136/jnnp-2014-309857, indexed in Pubmed: 25632155.

69. Werring DJ, Charidimou A, Charidimou A, et al. authors. Microbleeds, cerebral hemorrhage, and functional outcome after stroke thrombolysis. Stroke. 2017; 48(8): 2084-2090, doi: 10.1161/STROKEAHA.116.012992, indexed in Pubmed: 28720659.

70. Shoamanesh A, Kwok CS, Lim PA, et al. Postthrombolysis intracranial hemorrhage risk of cerebral microbleeds in acute stroke patients: a systematic review and meta-analysis. Int J Stroke. 2013; 8(5): 348-356, doi: 10.1111/j.1747-4949.2012.00869.x, indexed in Pubmed: 22973896.

71. Kakuda W, Thijs VN, Lansberg MG, et al. DEFUSE Investigators. R Bammer, L Wechsler, S Kemp, Difuse Investigator. Clinical importance of microbleeds in patients receiving IV thrombolysis. Neurology. 2005; 65(98): 1175-1178, doi: 10.1212/01. wnl.0000180519.27680.0f, indexed in Pubmed: 16247042.

72. Zand R, Tsivgoulis G, Singh M, et al. Safety of intravenous thrombolysis among stroke patients taking new oral anticoagulants-case series and systematic review of reported cases. J Stroke Cerebrovasc Dis. 2015; 24(12): 2685-2693, doi: 10.1016/j.jstrokecerebrovasdis.2015.07.021, indexed in Pubmed: 26542821.

73. Tsivgoulis G, Zand R, Katsanos AH, et al. Risk of symptomatic intracerebral hemorrhage after intravenous thrombolysis in patients with acute ischemic stroke and high cerebral microbleed burden: A metaanalysis. JAMA Neurol. 2016; 73(6): 675-683, doi: 10.1001/jamaneurol.2016.0292, indexed in Pubmed: 27088650.

74. Yan J, Qiu J, Wu X, et al. Pretreatment cerebral microbleeds and symptomatic intracerebral hemorrhage post-thrombolysis: a systematic review and meta-analysis. J Neurol. 2020; 267(2): 301307, doi: 10.1007/s00415-018-9156-5, indexed in Pubmed: 30542950.

75. Choi KH, Kim JH, Kang KW, et al. Impact of microbleeds on outcome following recanalization in patients with acute ischemic stroke. Stroke. 2018 [Epub ahead of print]: STROKEAHA118023084, doi: 10.1161/ STROKEAHA.118.023084, indexed in Pubmed: 30580721.

76. Shi ZS, Duckwiler GR, Jahan R, et al. Mechanical thrombectomy for acute ischemic stroke with cerebral microbleeds. J Neurointerv Surg. 2016; 8(6): 563-567, doi: 10.1136/neurintsurg-2015-011765, indexed in Pubmed: 25994939.

77. Wu X, Yan J, Ye H, et al. Pre-treatment cerebral microbleeds and intracranial hemorrhage in patients with ischemic stroke receiving endovascular therapy: a systematic review and meta-analysis. J Neurol. 2020; 267(5): 1227-1232, doi: 10.1007/s00415-019-09210-6, indexed in Pubmed: 30689017.

78. Kakumoto K, Matsumoto S, Nakahara I, et al. Rapid formation of cerebral microbleeds after carotid artery stenting. Cerebrovasc Dis Extra. 2012; 2(1): 9-16, doi: 10.1159/000337143, indexed in Pubmed: 22566991. 
79. Ito AO, Shindo A, li Y, et al. Microbleeds after carotid artery stenting: small embolism may induce cerebral microbleeds. Cerebrovasc Dis Extra. 2019; 9(2): 57-65, doi: 10.1159/000500112, indexed in Pubmed: 31203282.

80. Müller MD, Jongen LM, Altinbas A, et al. ICSS-MRI Study Investigators. Silent intracerebral hemorrhage in patients randomized to stenting or endarterectomy for symptomatic carotid stenosis. J Stroke. 2019; 21(1): 116-119, doi: 10.5853/jos.2018.02838, indexed in Pubmed: 30732448.

81. Liu S, Li C. Antiplatelet drug use and cerebral microbleeds: A metaanalysis of published studies. J Stroke Cerebrovasc Dis. 2015; 24(10): 2236-2244, doi: 10.1016/j.jstrokecerebrovasdis.2015.05.022, indexed in Pubmed: 26272868.

82. Lovelock CE, Cordonnier C, Naka H, et al. Edinburgh Study Group. Antithrombotic drug use, cerebral microbleeds, and intracerebral hemorrhage: a systematic review of published and unpublished studies. Stroke. 2010; 41(6): 1222-1228, doi: 10.1161/STROKEAHA.109.572594, indexed in Pubmed: 20431083.

83. Wang DN, Hou XW, Yang BW, et al. Quantity of cerebral microbleeds, antiplatelet therapy, and intracerebral hemorrhage outcomes: A systematic review and meta-analysis. J Stroke Cerebrovasc Dis. 2015; 24(12): 2728-2737, doi: 10.1016/j.jstrokecerebrovasdis.2015.08.003, indexed in Pubmed: 26342996.

84. Qiu J, Ye H, Wang J, et al. Antiplatelet therapy, cerebral microbleeds, and intracerebral hemorrhage: A meta-analysis. Stroke. 2018; 49(7): 1751-1754, doi: 10.1161/STROKEAHA.118.021789, indexed in Pubmed: 29798835.

85. Ge L, Ouyang X, Ban C, et al. Cerebral microbleeds in patients with ischemic cerebrovascular disease taking aspirin or clopidogrel. Medicine (Baltimore). 2019; 98(9): e14685, doi: 10.1097/ MD.0000000000014685, indexed in Pubmed: 30817601.

86. Rockson SG, Albers GW. Comparing the guidelines: anticoagulation therapy to optimize stroke prevention in patients with atrial fibrillation. J Am Coll Cardiol. 2004; 43(6): 929-935, doi: 10.1016/j. jacc.2003.11.028, indexed in Pubmed: 15028346.

87. Lioutas VA, Goyal N, Katsanos AH, et al. Clinical outcomes and neuroimaging profiles in nondisabled patients with anticoagulant-related intracerebral hemorrhage. Stroke. 2018; 49(10): 2309-2316, doi: 10.1161/ STROKEAHA.118.021979, indexed in Pubmed: 30355114.

88. Wilson D, Seiffge DJ, Traenka C, et al. And the CROMIS-2 collaborators. Outcome of intracerebral hemorrhage associated with different oral anticoagulants. Neurology. 2017; 88(18): 1693-1700, doi: 10.1212/ WNL.0000000000003886, indexed in Pubmed: 28381513.

89. Purrucker JC, Wolf M, Haas $\mathrm{K}$, et al. the RASUNOA Investigators. Microbleeds in ischemic vs hemorrhagic strokes on novel oral anticoagulants. Acta Neurol Scand. 2018; 138(2): 163-169, doi: 10.1111/ ane.12934, indexed in Pubmed: 29663313.

90. Lioutas VA, Goyal N, Katsanos AH, et al. Microbleed prevalence and burden in anticoagulant-associated intracerebral bleed. Ann Clin TransI Neurol. 2019; 6(8): 1546-1551, doi: 10.1002/acn3.50834, indexed in Pubmed: 31402613.
91. Wilson D, Ambler G, Shakeshaft C, et al. CROMIS-2 collaborators. Cerebral microbleeds and intracranial haemorrhage risk in patients anticoagulated for atrial fibrillation after acute ischaemic stroke or transient ischaemic attack (CROMIS-2): a multicentre observational cohort study [published correction appears in 2018; 1796): 578]. Lancet Neurol. 2018; 17(6): 539-547, doi: 10.1016/S14744422(18)30145-5, indexed in Pubmed: 29778365.

92. Charidimou A, Karayiannis C, Song TJ, et al. International META-MICROBLEEDS Initiative. Brain microbleeds, anticoagulation, and hemorrhage risk: Meta-analysis in stroke patients with AF. Neurology. 2017; 89(23): 2317-2326, doi: 10.1212/WNL.0000000000004704, indexed in Pubmed: 29117953.

93. Haussen DC, Henninger N, Kumar S, et al. Statin use and microbleeds in patients with spontaneous intracerebral hemorrhage. Stroke. 2012; 43(10): 2677-2681, doi: 10.1161/STROKEAHA.112.657486, indexed in Pubmed: 22829545.

94. Ziff OJ, Banerjee G, Ambler G, et al. Statins and the risk of intracerebral haemorrhage in patients with stroke: systematic review and metaanalysis. J Neurol Neurosurg Psychiatry. 2019; 90(1): 75-83, doi: 10.1136/jnnp-2018-318483, indexed in Pubmed: 30150320.

95. Wieberdink RG, Poels MMF, Vernooij MW, et al. Serum lipid levels and the risk of intracerebral hemorrhage: the Rotterdam Study. Arterioscler Thromb Vasc Biol. 2011; 31(12): 2982-2989, doi: 10.1161/ ATVBAHA.111.234948, indexed in Pubmed: 21921260.

96. Katsanos AH, Lioutas VA, Charidimou A, et al. International META-MICROBLEEDS Initiative. Statin treatment and cerebral microbleeds: A systematic review and meta-analysis. J Neurol Sci. 2021; 420: 117224, doi: 10.1016/j.jns.2020.117224, indexed in Pubmed: 33183779.

97. Demaerschalk BM, Kleindorfer DO, Adeoye OM, et al. American Heart Association Stroke Council and Council on Epidemiology and Prevention. Scientific rationale for the inclusion and exclusion criteria for intravenous alteplase in acute ischemic stroke: A statement for healthcare professionals from the American Heart Association/American Stroke Association. Stroke. 2016; 47(2): 581-641, doi: 10.1161/ STR.0000000000000086, indexed in Pubmed: 26696642.

98. Smith EE, Saposnik G, Biessels GJ, et al. American Heart Association Stroke Council; Council on Cardiovascular Radiology and Intervention; Council on Functional Genomics and Translational Biology; and Council on Hypertension. Prevention of stroke in patients with silent cerebrovascular disease: A scientific statement for healthcare professionals from the American Heart Association/American Stroke Association. Stroke. 2017; 48(2): e44-e71, doi: 10.1161/ STR.0000000000000116, indexed in Pubmed: 27980126.

99. Ahmed N, Audebert H, Turc G, et al. Consensus statements and recommendations from the ESO-Karolinska Stroke Update Conference, Stockholm 11-13 November 2018. Eur Stroke J. 2019; 4(4): 307-317, doi: 10.1177/2396987319863606, indexed in Pubmed: 31903429.

100. Canavero I, Micieli G, Paciaroni M. Decision algorithms for direct oral anticoagulant use in patients with nonvalvular atrial fibrillation: A practical guide for neurologists. Clin Appl Thromb Hemost. 2018; 24(3): 396-404, doi: 10.1177/1076029617720068, indexed in Pubmed: 28914077. 